\title{
Preparation of Colloidal Dispersions of Graphene Sheets in Organic Solvents by Using Ball Milling
}

\author{
Weifeng Zhao, Furong Wu, Hang Wu, and Guohua Chen \\ Institute of Polymer \& Nanomaterials, Huaqiao University, Quanzhou, Fujian 362021, China \\ Correspondence should be addressed to Guohua Chen, hdcgh@hqu.edu.cn
}

Received 1 June 2010; Accepted 8 July 2010

Academic Editor: Rakesh Joshi

Copyright () 2010 Weifeng Zhao et al. This is an open access article distributed under the Creative Commons Attribution License, which permits unrestricted use, distribution, and reproduction in any medium, provided the original work is properly cited.

\begin{abstract}
A top-down method was developed for producing colloidal dispersions of graphene sheets. Graphite nanosheets comprising hundreds of carbon layers were dispersed and gently ball-milled to exfoliate into graphene in a variety of organic solvents. After 30 hours of the shear-force-dominated grinding and a subsequent 4000 r.p.m. of centrifugation, single- and few-layer graphene sheets were readily prepared and homogeneously and stably suspended in the good solvent medium which possesses a surface tension value close to $40 \mathrm{~mJ} \mathrm{~m}^{-2}$, such as in $N, N$-dimethylformamide, at a concentration up to $0.08 \mathrm{mg} \mathrm{ml}^{-1}$, achieving a yield higher than $32.0 \mathrm{wt} \%$. The graphene materials in the colloidal suspension were characterized using scanning and transmission electron microscopy and atomic force microscopy.
\end{abstract}

\section{Introduction}

With a strictly 2D structure and novel electronic and mechanical properties, graphene has rapidly become an amazing form of carbon that may be used as active components in a wide range of applications such as field-effect transistors [1], transparent conductors [2], liquid crystal displays [3], electromechanical resonators [4], composites [5], energy storage, and photovoltaic devices [6, 7]. To accelerate the use of graphene and tailor its properties, homogeneous colloidal suspensions of individual graphene sheets are always expected, for various processing of the graphene materials such as film and paper depositions, surface modifications and functionalization chemistry of the edges, and defects of graphene sheets are preferred to be conducted in the liquid phase. The key point for creating graphene suspension is overcoming the enormous van der Waals-like forces between graphite layers to yield a complete exfoliation of graphite flakes and dispersing the resulted graphene sheets stably in a liquid media.

In recent years, sonication has been extensively used as an exfoliation and dispersion strategy to produce colloidal suspensions of graphene sheets in a liquid phase [8-11]. The starting materials vary from pristine graphite to graphite derivatives (e.g., graphite oxide $[12,13])$. This procedure has been successful in various solvents with a surface tension value $40-50 \mathrm{~mJ} \mathrm{~m}^{-2}$ which are good media for graphite exfoliation $[8,14]$, especially with the aid of a third, dispersant phase, such as surfactants and polymers $[9,10]$. However, for the dispersions of unfunctionalized graphene sheets without surfactants or stabilizers in common solvents, the concentrations attainable are still low $\left(\leq 0.03 \mathrm{mg} \mathrm{ml}^{-1}\right)$ $[8,14]$. This might be probably due to some limitations of sonication in graphite exfoliation. In fact, it has been found that excessive sonication cannot lead to higher yield of graphenes but destruct the graphene sheets $[8,9]$. Herein, ball-milling was used to exfoliate graphite in a wide variety of organic solvents including ethanol, formamide, acetone, tetrahydrofuran(THF), tetramethyluren(TMU), $\mathrm{N}, \mathrm{N}$-dimethylformamide(DMF), and $\mathrm{N}$-methylpyrrolidone (NMP) to create colloidal dispersions of unfuctionalized graphene sheets. The wet ball-milling experiments were carried out in a planetary mill which mainly exerts shear forces on the materials [15]. Moreover, the rotating tray of the planetary mill was controlled at a low speed to further limit the shock forces applied by the ball impact. Due to the weak van der Waals-like coupling between graphite layers, the graphene sheets in graphite can slide easily with respect to one another under the applied shear forces, creating 


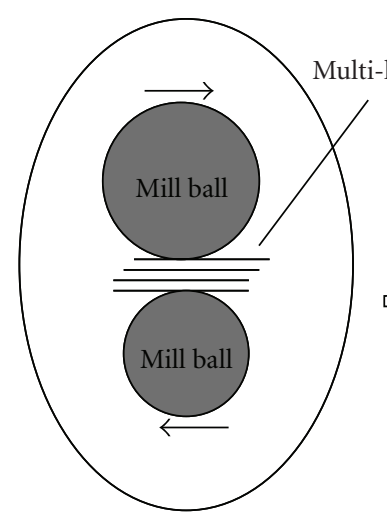

(a)

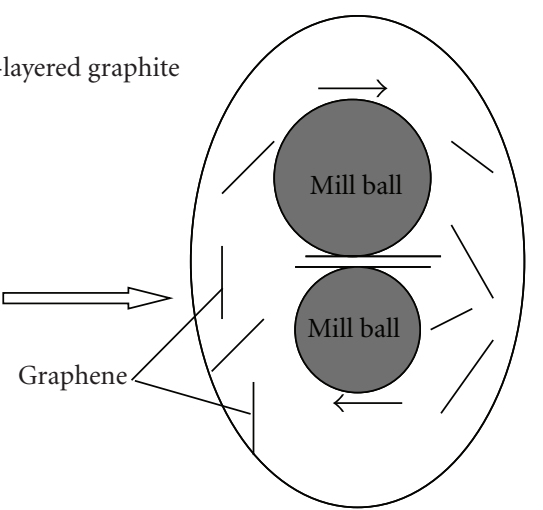

(b)

FIGURE 1: Schematic illustration for the creation of graphene from multilayered graphite by using wet ball-milling: (a) GNs dispersed in solvent are delaminated by mill balls in grinding process; (b) graphene sheets are formed in situ in solvent. The arrows indicate the shear forces applied to graphite platelets.

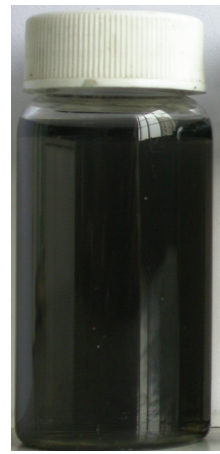

(a)

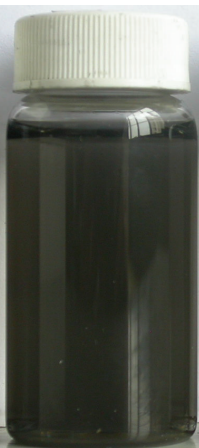

(b)

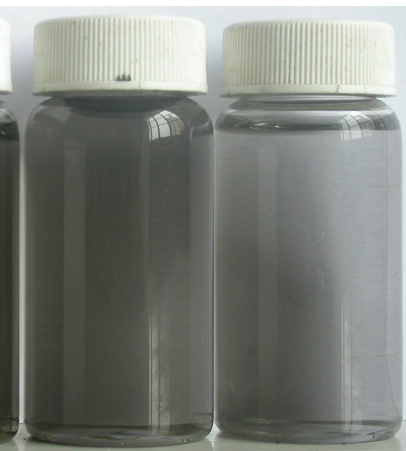

(c)

(d)
FIGURE 2: Dispersions of graphene materials in TMU (a), NMP (b), DMF (c), THF (d) after $4000 \mathrm{r} / \mathrm{min}$ of centrifugation. All of the samples have been kept quietly for six months.

isolated graphene sheets in the solvent media in situ, as illustrated in Figure 1.

\section{Experimental}

2.1. Preparation Procedure. The starting material, graphite nanosheets (GNs), was prepared by sonicating expanded graphite in an ethanol solution of water as reported in the literature [16]. All the organic solvents are of AR grade. In a typical procedure for wet grinding, $0.02 \mathrm{~g}$ of dried GNs were dispersed in $80 \mathrm{~mL}$ of an organic solvent (anhydrous DMF, e.g.) by a mechanical stirring, introduced with zirconia balls $(2.0-2.5 \mathrm{~mm}$ in diameter, $200 \mathrm{~g}$ of weight) into a poly(tetrafluoroethylene) vial, and milled in a planetary mill at a mild rotation speed 300 r. p.m. to ensure the shear stress is dominant. After ball-milling for 30 hours, the obtained dark dispersion was placed quietly to let large pieces subside completely, and then was subjected to centrifugation at 4000 r.p.m. for $30 \mathrm{~min}$ to remove any macroscopic aggregates using a TGL-10B centrifuge, giving a black suspension with

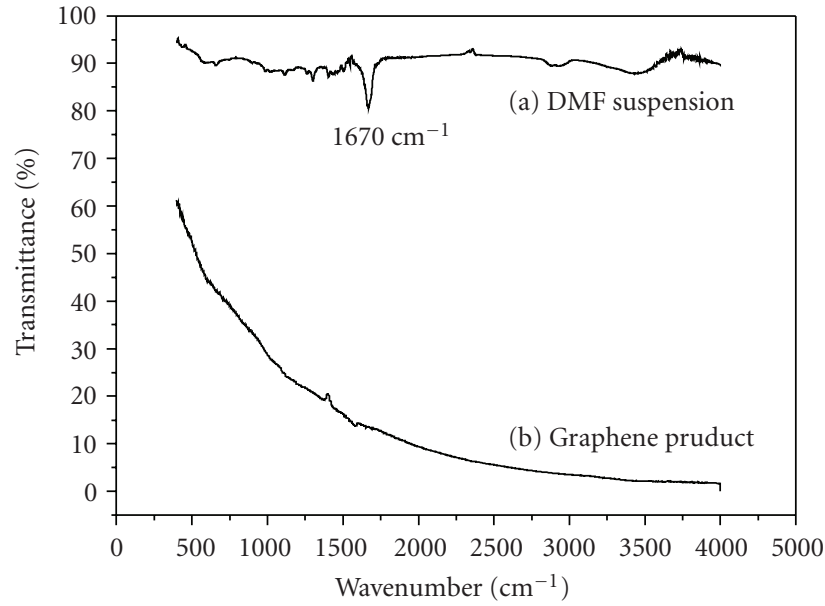

FIGURE 3: FTIR spectra for DMF-based graphene suspension (a) and dried graphene product (b).

single- and few-layer graphene sheets stably suspended in the good solvent (Figure 2.).

2.2. Characterization. Absorbance was recorded on a UVvisible spectrophotometer (UV-1600, China) using a quartz cell with a path length of $10 \mathrm{~mm}$ at room temperature. Transmission electron microscopy (TEM) was performed on a JEM-2010 JEOL transmission electron microscope at an accelerating voltage of $300 \mathrm{kV}$. TEM specimens were prepared by either drop-casting graphenes from the supernatant or loading the microtomed epoxy composite slices of the asobtained graphene powder onto copper grids coated with carbon film. Images of scanning electron microscopy (SEM) were acquired on graphene powder without golden coating using a LEO 1530 field-emitting scanning electron microscope (Germany) at the operating voltage of $20 \mathrm{KV}$. Fourier transform infrared spectroscopy (FTIR) of was recorded from $\mathrm{KBr}$ pellets on a FTIR-84005 spectrometer (Japan). 


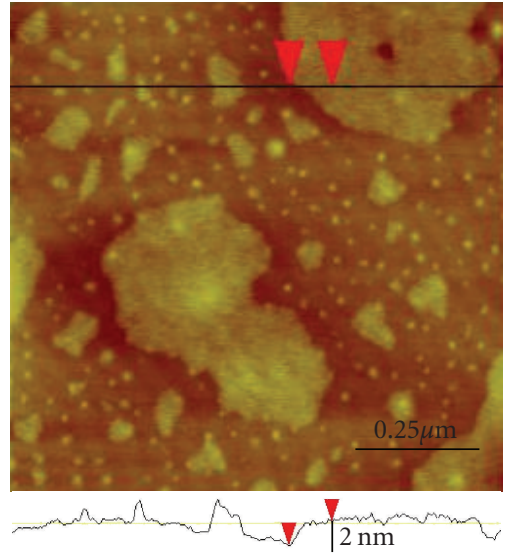

(a)

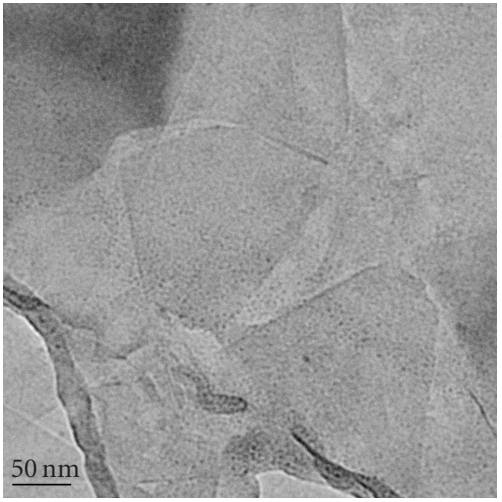

(c)

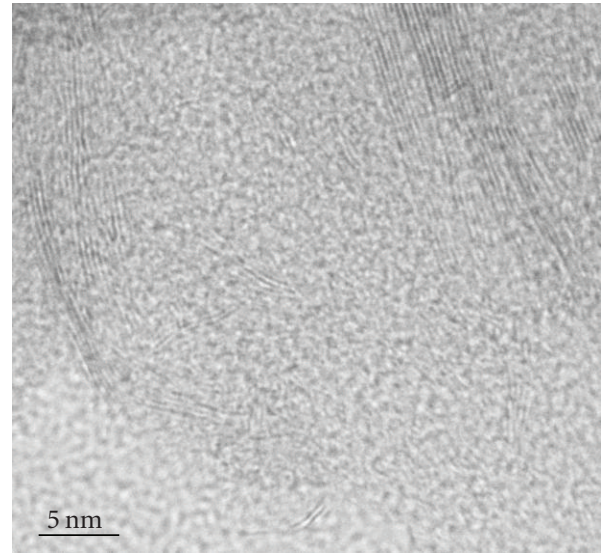

(b)

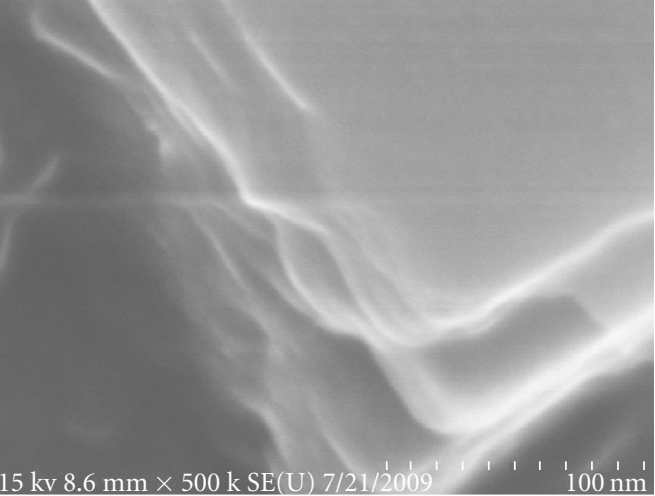

(d)

Figure 4: Supernatant graphene sheets in DMF. (a) AFM image of the supernatant graphene deposited on a mica substrate, followed with the corresponding height cross-section along the line in the image; the height difference between red arrows is $\sim 1.8 \mathrm{~nm}$. (b, c) HRTEM image of separated graphene sheets embedded in polymer slice (b) and isolated flat graphene flakes solution-dropped on TEM grid, from which scrolled edges were observed (c). (d) SEM images of restacked graphene sheets forming a paper-like layered structure.

\section{Results and Discussion}

Our starting material, GNs, has a nanoscale thickness ranging from 30 to $80 \mathrm{~nm}$, while the lateral size appears much larger, which is of $5-20 \mu \mathrm{m}$. The features of nanometer sized thickness and outstanding surface area are advanced in improving the solvent-graphene layer interaction, should make the GNs exfoliate into few- even single-layered graphenes with great efficiency and speed.

The shear-force-type wet ball-milling/4000 r.p.m of centrifugation creates a homogeneous, highly stable, dark suspension of graphene in the good solvents as shown in Figure 2. What is notable is that the as-prepared dispersions are very stable at room temperature; sedimentation can hardly occur within nearly three weeks of quiet placement except for slight aggregations. The agglomerated graphene particles remaining well suspended in solvent could be readily re-dispersed by a simple diluting, for a high concentration will lead to gelation over time. Furthermore, in a low concentration suspension, no aggregations were observed after being placed quietly for long periods of time (at least six months), indicating an entire dispersion of individual graphene sheets. After evaporating solvent under vacuum and repeated washing with ethanol, dried powder sample with a high purity were obtained for characterizations and for plotting standard curves of absorption. As shown in Figure 3, no DMF absorption features are observed in the graphene product FTIR spectrum which appears featureless, indicating a complete removal of the residual organics by repeated washing.

Figure 4(a) displays the typical AFM image of isolated graphene sheets evaporated from their dilute dispersion on freshly cleaved mica support. Topographic heights of the sheets estimated from the cross-sectional profile were found in the range of $0.8 \sim 1.8 \mathrm{~nm}$, an indicative of single- and few-layer graphenes. High-resolution transmission electron microscopy (HRTEM) is also a powerful technique used extensively to provide definitive identification of graphene materials. The cross-sections of graphene sheet show predominantly one line for a single-layer graphene, and several condensed dark lines for a few-layer sheet. HRTEM images of the supernatant materials in slice clearly give their crosssections by a direct visualization (Figure 4(b)). Individual one dark lines with a thickness $\sim 0.5 \mathrm{~nm}$ (corresponding to single atom carbon layers) can be readily identified. Fewlayer graphene sheets composing two or several dark lines 
TABLE 1: Fraction of graphene remaining after centrifugation.

\begin{tabular}{|c|c|c|c|}
\hline \multirow{2}{*}{ Solvent } & \multirow{2}{*}{ Surface tension $\left(\mathrm{mJ} \mathrm{m}^{-2}\right)$} & \multicolumn{2}{|c|}{ Remaining after 4000 r.p.m. of centrifugation } \\
\hline & & Concentration $(\mu \mathrm{g} / \mathrm{ml})$ & $\mathrm{wt} \%$ remaining \\
\hline NMP & 41 & 88.9 & 35.56 \\
\hline TMU & 34.7 & 88.28 & 35.31 \\
\hline DMF & 35.2 & 96.67 & 38.67 \\
\hline THF & 26.4 & 76.03 & 30.41 \\
\hline Acetone & 23.7 & 66.6 & 26.64 \\
\hline Ethanol & 22.27 & 10.32 & 4.128 \\
\hline Formamide & 58.35 & 3.67 & 1.468 \\
\hline
\end{tabular}

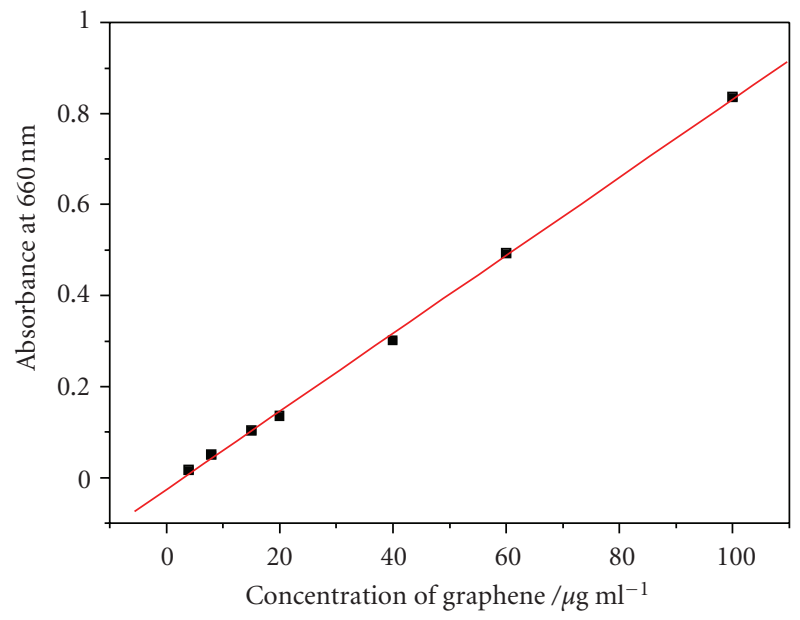

Figure 5: Plot of optical density at $660 \mathrm{~nm}$ versus graphene concentration. The straight line is a linear least squares fit of the experimental points.

were observed. Typically flat sheets deposited onto grid show a lateral size about $100-200 \mathrm{~nm}$ which is similar to that observed from the AFM image, and scroll on edges at times (Figure 4(c)). Due to the high-specific surface area, the graphene sheets have a significant tendency to restack, forming a paper-like layered structure when the solvent was evaporated (Figure 4(d)).

The good stability of the graphene suspensions enables the weight content of supernatant graphenes to be monitored using UV-vis spectroscopy, providing a reliable way to determine the graphene yield. As such, the as-prepared purified graphene samples from DMF were carefully weighted and readily redispersed in NMP with an agitation and a followed sonication of less than $1 \mathrm{~min}$, giving a stable and homogeneous dispersion. The absorption spectra of the graphene NMP suspensions with different concentrations were then measured. It was found that the absorbance was well in line with the relative graphene contents in NMP, obeying Beer's law perfectly in the given range of concentration. By plotting the optical absorption values at $660 \mathrm{~nm}$ of wavelength versus concentrations, a standard curve was generated, with an $R$ value of 0.999 (Figure 5.). According to Beer's law, the extinction coefficient was determined to be $1020 \mathrm{Lg}^{-1} \mathrm{~m}^{-1}$. Linear relationship between the absorbance and the relative concentrations of graphene at other wavelength, $270 \mathrm{~nm}$ for example, has also been observed, which strictly follows Beer's law too. The results suggest that the graphene samples had been re-dispersed well in NMP medium before the absorption measurements.

By measuring the absorbance of the as-centrifuged graphene suspensions at $660 \mathrm{~nm}$ wavelength which matches the standard curve, the concentration of the supernatant graphene could then be calculated. Table 1 lists the contents of graphene remaining in solvents of different surface tensions after centrifugation at 4000 r.p.m. According to literatures $[8,17,18]$, the values of surface tensions of graphene layers close to $40 \mathrm{~mJ} \mathrm{~m}^{-2}$. In the good solvents with the surface tensions matches that of graphene sheets, such as DMF, NMP, and TMU, the graphene concentrations up to $0.08 \mathrm{mg} \mathrm{ml}^{-1}$ are significant higher compared with the case of ethanol and formamide where less than $5.0 \%$ of the materials remained. Colloidal suspension of single- and fewlayer graphene sheets with a concentration $\sim 0.096 \mathrm{mg} \mathrm{ml}^{-1}$ has been achieved in DMF, and the mass fraction remaining after centrifugation - which is also the yield for graphenereaches higher than $38.0 \mathrm{wt} \%$.

\section{Conclusions}

An effective method for creating colloidal suspensions of graphene sheets without the use of surfactants/stabilizers was proposed via exfoliating GNs in organic liquid media using wet ball-milling process. In the good solvents for graphite exfoliation including DMF, NMP, and TMU, suspensions of sigle- and few-layer graphene sheets can be produced facilely at a significantly high yield without any recycling. The ability to provide stable suspensions of graphene sheets in organic solvents with such a high concentration should facilitate the processing of graphene materials remarkably. We believe that either theoretical investigations or practical applications related to graphene, such as functionalization chemistry of the sheet edges, and graphene surface modification will benefit a lot from this bulk exfoliation technique.

\section{Acknowledgments}

This work was funded by National Natural Science Foundation of China (nos. 20574025, 50373015) and Natural Science Foundation of Fujian Province (no. E0820001). 


\section{References}

[1] B. Obradovic, R. Kotlyar, F. Heinz et al., "Analysis of graphene nanoribbons as a channel material for field-effect transistors," Applied Physics Letters, vol. 88, no. 14, Article ID 142102, 2006.

[2] G. Eda, G. Fanchini, and M. Chhowalla, "Large-area ultrathin films of reduced graphene oxide as a transparent and flexible electronic material," Nature Nanotechnology, vol. 3, no. 5, pp. 270-274, 2008.

[3] P. Blake, P. D. Brimicombe, R. R. Nair et al., "Graphene-based liquid crystal device," Nano Letters, vol. 8, no. 6, pp. 17041708, 2008.

[4] J. S. Bunch, A. M. van der Zande, S. S. Verbridge et al., "Electromechanical resonators from graphene sheets," Science, vol. 315, no. 5811, pp. 490-493, 2007.

[5] S. Stankovich, D. A. Dikin, G. H. B. Dommett et al., "Graphene-based composite materials," Nature, vol. 442, no. 7100, pp. 282-286, 2006.

[6] M. D. Stoller, S. Park, Y. Zhu, J. An, and R. S. Ruoff, "Graphene-based ultracapacitors," Nano Letters, vol. 8, no. 10, pp. 3498-3502, 2008.

[7] Z. Liu, Q. Liu, Y. Huang et al., "Organic photovoltaic devices based on a novel acceptor material: graphene," Advanced Materials, vol. 20, no. 20, pp. 3924-3930, 2008.

[8] Y. Hernandez, V. Nicolosi, M. Lotya et al., "High-yield production of graphene by liquid-phase exfoliation of graphite," Nature Nanotechnology, vol. 3, no. 9, pp. 563-568, 2008.

[9] X. Li, X. Wang, L. Zhang, S. Lee, and H. Dai, "Chemically derived, ultrasmooth graphene nanoribbon semiconductors," Science, vol. 319, no. 5867, pp. 1229-1232, 2008.

[10] X. Li, G. Zhang, X. Bai et al., "Highly conducting graphene sheets and Langmuir-Blodgett films," Nature Nanotechnology, vol. 3, no. 9, pp. 538-542, 2008.

[11] W. Gu, W. Zhang, X. Li et al., "Graphene sheets from wormlike exfoliated graphite," Journal of Materials Chemistry, vol. 19, no. 21, pp. 3367-3369, 2009.

[12] S. Park, J. An, I. Jung et al., "Colloidal suspensions of highly reduced graphene oxide in a wide variety of organic solvents," Nano Letters, vol. 9, no. 4, pp. 1593-1597, 2009.

[13] S. Stankovich, D. A. Dikin, R. D. Piner et al., "Synthesis of graphene-based nanosheets via chemical reduction of exfoliated graphite oxide," Carbon, vol. 45, no. 7, pp. 1558$1565,2007$.

[14] C. E. Hamilton, J. R. Lomeda, Z. Sun, J. M. Tour, and A. R. Barron, "High-yield organic dispersions of unfunctionalized graphene," Nano Letters, vol. 9, no. 10, pp. 3460-3462, 2009.

[15] M. V. Antisari, A. Montone, N. Jovic, E. Piscopiello, C. Alvani, and L. Pilloni, "Low energy pure shear milling: a method for the preparation of graphite nano-sheets," Scripta Materialia, vol. 55, no. 11, pp. 1047-1050, 2006.

[16] G. Chen, D. Wu, W. Weng, and C. Wu, "Exfoliation of graphite flake and its nanocomposites," Carbon, vol. 41, no. 3, pp. 619621, 2003.

[17] S. D. Bergin, V. Nicolosi, P. V. Streich et al., "Towards solutions of single-walled carbon nanotubes in common solvents," Advanced Materials, vol. 20, no. 10, pp. 1876-1881, 2008.

[18] S. D. Bergin, Z. Sun, D. Rickard, P. V. Streich, J. P. Hamilton, and J. N. Coleman, "Multicomponent solubility parameters for single-walled carbon nanotube-solvent mixtures," ACS Nano, vol. 3, no. 8, pp. 2340-2350, 2009. 

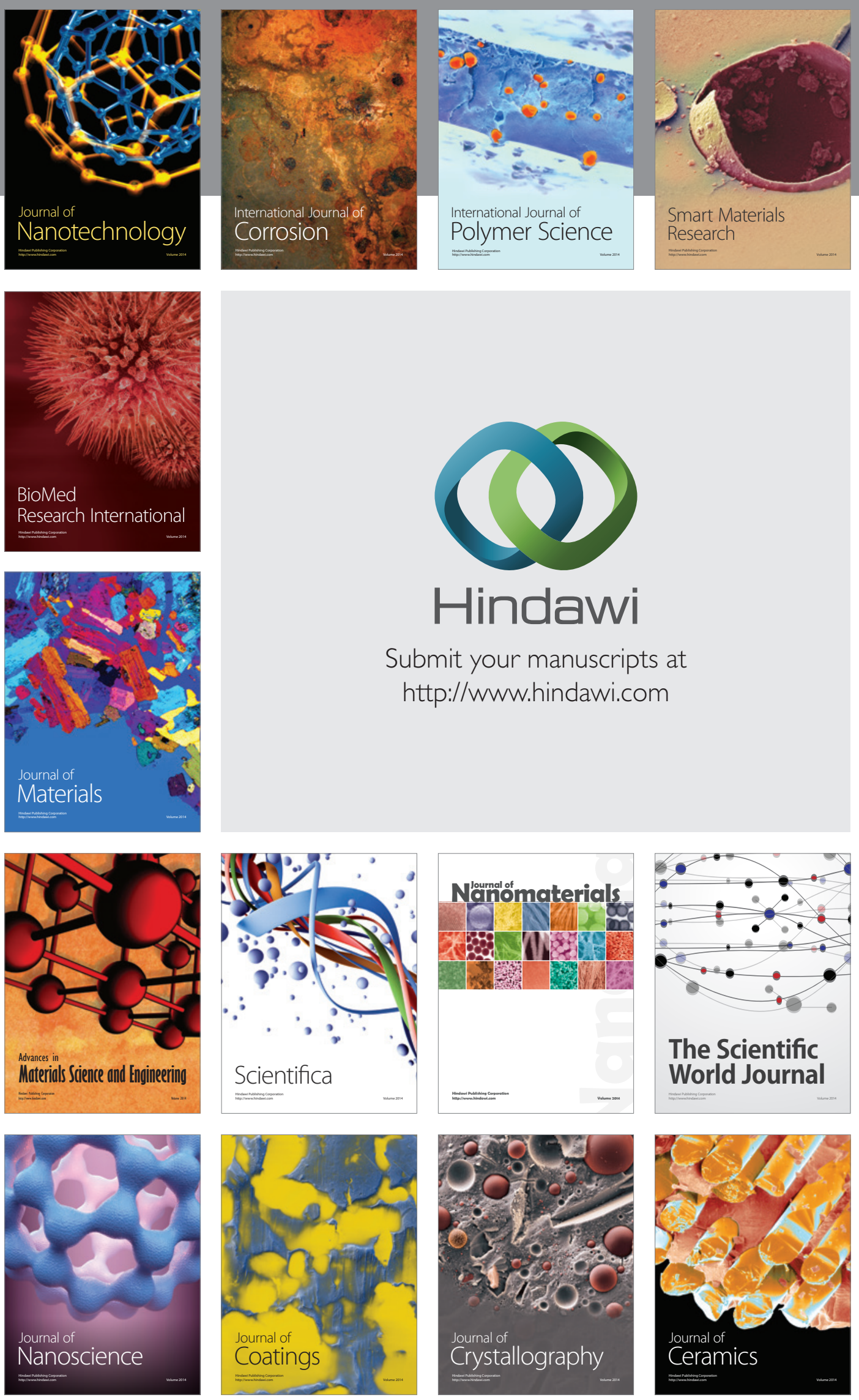

The Scientific World Journal

Submit your manuscripts at

http://www.hindawi.com

\section{World Journal}

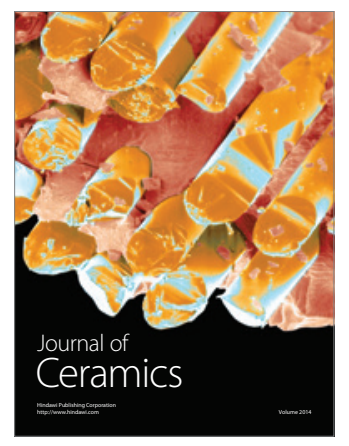

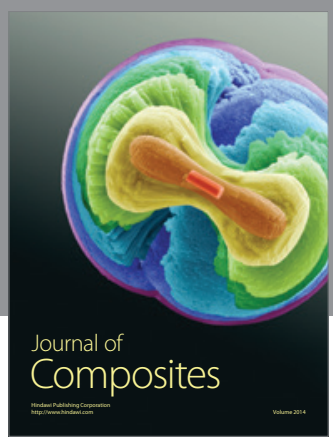
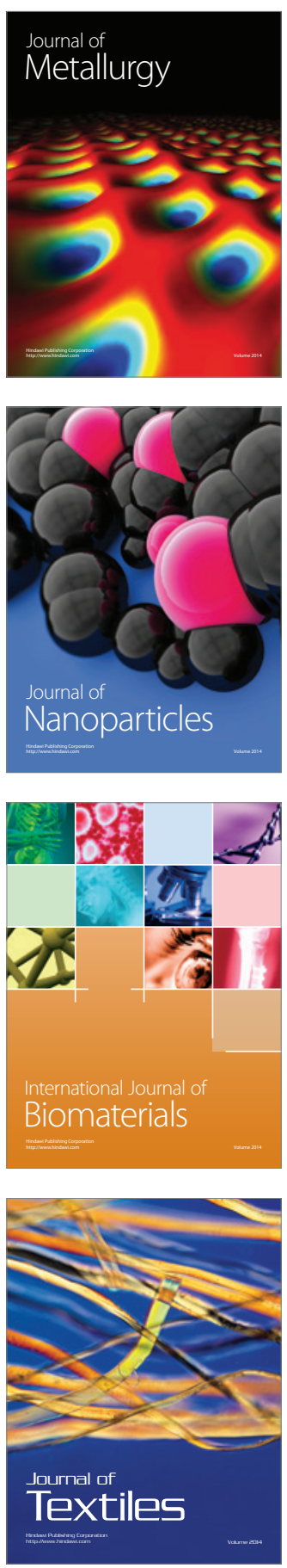